\title{
The Effect of Soft and Rigid Cervical Collars on Head and Neck Immobilization in Healthy Subjects
}

\author{
Kourosh Barati ${ }^{1}$, Mokhtar Arazpour $^{2}$, Roshanak Vameghi ${ }^{3}$, Ali Abdoli ${ }^{4}$, Farzad Farmani ${ }^{5}$ \\ ${ }^{1}$ Department of Orthotics and Prosthetics, School of Rehabilitation Sciences, Iran University of Medical Sciences, Tehran, Iran \\ ${ }^{2}$ Department of Orthotics and Prosthetics, University of Social Welfare and Rehabilitation Sciences, Tehran, Iran \\ ${ }^{3}$ Pediatric Neurorehabilitation Research Center, University of Social Welfare and Rehabilitation Sciences, Tehran, Iran \\ ${ }^{4}$ Department of Neurosurgery, School of Medicine, Hamadan University of Medical Sciences, Hamadan, Iran \\ ${ }^{5}$ Department of Orthotics and Prosthetics, School of Rehabilitation Sciences, Hamadan University of Medical Sciences, Hamadan, Iran
}

Study Design: Whiplash injury is a prevalent and often destructive injury of the cervical column, which can lead to serious neck pain. Many approaches have been suggested for the treatment of whiplash injury, including anti-inflammatory drugs, manipulation, supervised exercise, and cervical collars. Cervical collars are generally divided into two groups: soft and rigid collars.

Purpose: The present study aimed to compare the effect of soft and rigid cervical collars on immobilizing head and neck motion.

Overview of Literature: Many studies have investigated the effect of collars on neck motion. Rigid collars have been shown to provide more immobilization in the sagittal and transverse planes compared with soft collars. However, according to some studies, soft and rigid collars provide the same range of motion in the frontal plane.

Methods: Twenty-nine healthy subjects aged 18-26 participated in this study. Data were collected using a three-dimensional motion analysis system and six infrared cameras. Eight markers, weighing $4.4 \mathrm{~g}$ and thickened $2 \mathrm{~cm}^{2}$ were used to record kinematic data. According to the normality of the data, a paired $t$-test was used for statistical analyses. The level of significance was set at $\alpha=0.01$.

Results: All motion significantly decreased when subjects used soft collars $(p<0.01)$. According to the obtained data, flexion and lateral rotation experienced the maximum (39\%) and minimum (11\%) immobilization in all six motions using soft collars. Rigid collars caused maximum immobilization in flexion (59\%) and minimum immobilization in the lateral rotation $(18 \%)$ and limited all motion much more than the soft collar.

Conclusions: This study showed that different cervical collars have different effects on neck motion. Rigid and soft cervical collars used in the present study limited the neck motion in both directions. Rigid collars contributed to significantly more immobilization in all directions.

Keywords: Whiplash injury; Collar; Immobilization; Neck; Head

\section{Introduction}

Whiplash injury is a prevalent and often destructive injury of the cervical column with an annual incidence of 235 per 100,000 people [1]. Whiplash injury leads to serious neck pain, with side effects remaining for 2 years postinjury in approximately $50 \%$ of patients.

Studies have shown that neck injury should be con-

\footnotetext{
Received Sep 13, 2016; Revised Dec 22, 2016; Accepted Dec 26, 2016

Corresponding author: Farzad Farmani

Department of Orthotics and Prosthetics, School of Rehabilitation Sciences, Hamadan University of Medical Sciences,

Shahid Fahmideh Street, Hamadan, Iran. Postal code: 6517838736

Tel: +98-81-38381571, Fax: +98 8138381572 , E-mail: farmani.farzad@yahoo.com
} 
trolled and treated quickly to avoid further injury. Many approaches have been suggested to manage whiplash injury, including anti-inflammatory drugs, manipulation, supervised exercise, and cervical collars.

In many cases, no clinical signs present immediately after injury to the cervical column. Therefore, neck immobilization using cervical collars, even before radiographic evaluation has been completed, is a standard treatment approach for patients suffering from multiple injuries. Using a cervical collar is the first intervention when encountering cervical column injuries.

Cervical collars are generally divided into two groups: soft and rigid collars. Routinely, soft collars are prescribed for whiplash injury; however, some studies have suggested the use of rigid collars [2,3]. The primary function of cervical collars is to immobilize the cervical spine [4]. Cervical collars are typically used for an extended period of time, and they should immobilize the head and neck movement while allowing for normal daily activities. Rigid collars provide more immobilization in the sagittal and transverse planes compared with soft collars. However, some studies have shown that soft and rigid collars both provide the same range of motion (ROM) in the frontal plane. Another study using a goniometer showed that the cervical column is limited more by rigid collars than by soft ones in all planes. Patient comfort is a critical factor, which encourages caregivers to give preference to soft collars in prescriptions.

Considering that both rigid and soft collars are prevalent in the treatment of whiplash injury, the present study aimed to compare the effects of soft and rigid cervical collars on immobilizing head and neck motion.

\section{Materials and Methods}

\section{Participants}

This semi-experimental study examined 29 healthy people aged 18-26 years without any history of neck surgery or orthopedic problems in the shoulders and spine. All participants signed an informed consent form. To calculate the sample size, considering a confidence level of $99 \%$ and power of $80 \%$, we used a similar study to calculate sample size. Table 1 shows demographic information of the participants. This study was approved by the medical ethics committee of University of Social Welfare and Rehabilitation Sciences.
Table 1. Demographic characterisics of the participants

\begin{tabular}{lcc} 
Characterisic & Standard deviation & Mean \\
Age & 1.56 & 20.9 \\
Weight & 10.1 & 63.9 \\
Height & 9.9 & 170 \\
\hline
\end{tabular}

\section{Intervention}

Soft and rigid cervical collars (Teb Sanat, Tehran, Iran) were tested in this study. The neck environment and length were used to determine the proper size of cervical collar for each participant, according to the manufacturer's instruction. Soft collars are flexible and composed of polyurethane foam rubber. It is easily wrapped around the neck and secured with a Velcro strap for support. Rigid collars are composed of hard polyethylene covered by a soft pad for patient comfort. It also had a Velcro strap in the back part of the neck for ease of removal (Figs. 1, 2).

\section{Equipment}

In the present study, data collection was performed using a three-dimensional (3D) motion analysis system (VI$\mathrm{CON}$ ) and six infrared cameras. Additionally, eight markers, weighing $4.4 \mathrm{~g}$ and thickened $2 \mathrm{~cm}^{2}$, were used and positioned on the seventh cervical vertebrae (one marker) below the jugular notch (one marker) on the right and left acromioclavicular joints (two markers) and at the anterior and posterior of the head (four markers). The markers were used to collect kinematic data [5,6]. Fig. 1 shows the positions of the markers. All tests were performed in the biomechanics laboratory of the University of Social Welfare and Rehabilitation.



Fig. 1. Soft cervical collar.

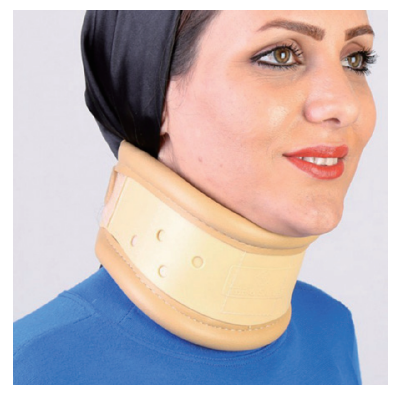

Fig. 2. Rigid cervical collar. 


\section{Measurements}

Measurements were performed in three conditions: no collar, soft collar, and rigid collar. To become familiar with the cervical collars, subjects were asked to wear

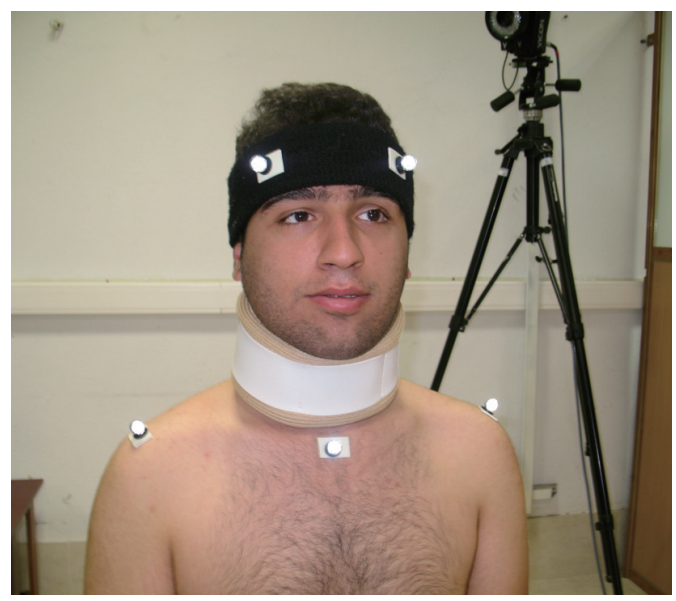

Fig. 3. Markers position (anterior view).

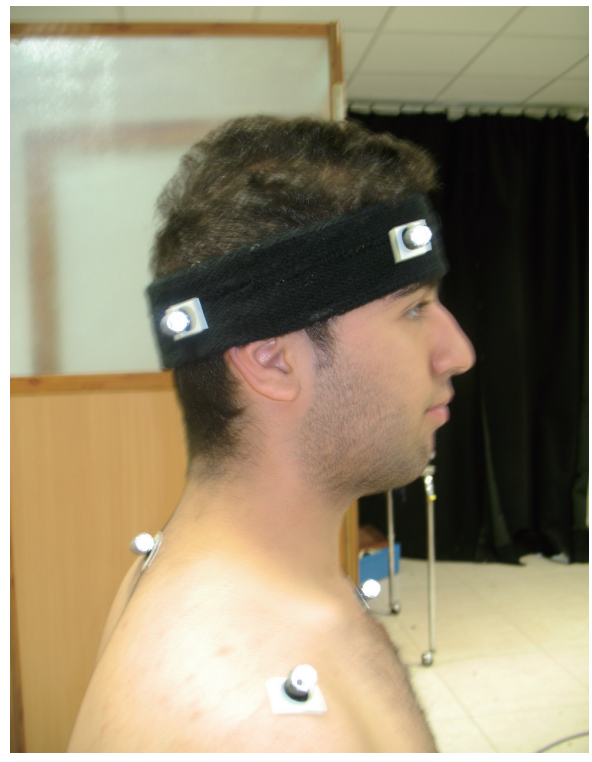

Fig. 4. Markers position (lateral view). the collars for 10-20 minutes before testing. They were instructed to perform six different neck motions including flexion, extension, rotation to the left and right, and lateral bending to the left and right using no collar and with collars. They wore the collars while sitting on a chair. Their head was in a neutral position facing anteriorly, focused on a point positioned at the level of the participant's eyes. All were asked to move the neck at a self-selected speed and force. Soft and rigid collars were utilized in a random sequence by each subject. During the test, all data obtained were recorded by a computer connected to the cameras (Figs. 3, 4).

\section{Data processing and analysis}

Data were analyzed using MATLAB (The Math-Works, Natick, MA, USA). The motion of the cervical region was calculated as a motion of the head relative to the upper body [5].

\section{Statistical analysis}

SPSS software ver. 16.0 (SPSS Inc., Chicago, IL, USA) was used to analyze data. Normality of the data was assessed using Kolmogorov-Smirnov test. According to the normality of the data, a paired $t$-test was utilized to analyze the effect of soft and rigid collars on the neck ROM in six different motions. The level of significance was set at $\alpha=0.01$.

\section{Results}

Table 2 provides information on the neck ROM with no collar and with soft and rigid collars. All motions significantly decreased when subjects used soft collars $(p<0.01)$. According to the data, flexion experienced maximum immobilization (39\%) and lateral rotation experienced the minimum immobilization (11\%) among all six motions

Table 2. Comparison of cervical spine ROM wearing soft and rigid collar using paired sample $t$-test

\begin{tabular}{lcccccc} 
Condition & Flexion & Extension & Rt. Lat. Flx & Lt. Lat. Flx & Rt. Lat. Rot & Lt. Lat. Rot \\
Soft collar & $31.3 \pm 2.7$ & $44.5 \pm 2.7$ & $34.9 \pm 1.6$ & $35 \pm 1.6$ & $56.8 \pm 1.3$ & $56.6 \pm 1.0$ \\
Rigid collar & $21 \pm 1.7$ & $33.7 \pm 2.8$ & $28.5 \pm 1.7$ & $28.6 \pm 2.0$ & $53.9 \pm 2.4$ & $53.5 \pm 2.0$ \\
$p$-value & $<0.01$ & $<0.01$ & $<0.01$ & $<0.01$ & $<0.01$ & $<0.01$ \\
\hline
\end{tabular}

Values are presented as mean \pm standard deviation.

ROM, range of motion; Rt. Lat. Flx, right lateral rotation; Lt. Lat. Flx, left lateral flexion; Rt. Rot, right lateral rotation; Lt. Lat. Rot., left lateral rotation. 
when soft collars were used. Similarly, a reduction of the neck ROM was significant in all motions when subjects used rigid collars $(p<0.01)$. Rigid collars caused maximum immobilization in flexion (59\%) and minimum immobilization in lateral rotation (18\%).

Table 3. Percentage of motion using soft and rigid collars

\begin{tabular}{lc} 
Motion/Collar type & Limitation percentage \\
Flexion & \\
Soft & 39 \\
\hline Rigid & 59 \\
Extension & \\
\hline Soft & 29 \\
\hline Rigid & 47 \\
\hline Rt. Lat. Flexion & \\
\hline Soft & 16 \\
\hline Rigid & 36 \\
\hline Lt. Lat. Flexion & \\
\hline Soft & 15 \\
\hline Rigid & 32 \\
\hline Rt. Lat. Rot & \\
\hline Soft & 11 \\
\hline Rigid & 18 \\
\hline Lt. Lat. Rot & 13 \\
\hline Soft & 18 \\
\hline Rigid & \\
\hline
\end{tabular}

Rt, right; Lt, left; Lat, lateral; Rot, rotation.
Table 3 shows the percentages of immobilization caused by soft and rigid collars in the six different motions. Rigid collars limited all motion much more than did soft collars. The minimum difference between soft and rigid collars in immobilizing the neck motion was related to the lateral rotation toward the right and left sides (Tables 3-5).

\section{Discussion}

The present study aimed to evaluate the effect of soft and rigid collars on head and neck immobilization. This study was performed using $3 \mathrm{D}$ motion capture to assess the head and neck ROM. This method is non-invasive and more reliable than other tests. This study was performed on young healthy participants without motion limitations.

For the neck ROM in subjects who were not using any cervical collar, $51^{\circ}$ of flexion, $61^{\circ}$ of extension, $41.5^{\circ}$ of lateral flexion to the right, $41.3^{\circ}$ of lateral flexion to the left, and $64.2^{\circ}$ of lateral rotation to the right and the left were observed. Due to the variety of methods used to measure ROM, it is difficult to compare our results with those of other studies. However, our results were similar to those reported by some previous studies [3,7-9].

The results of this study indicated that soft and rigid collars both limited head and neck ROM. This result is similar to those of previous studies $[3,10]$.

Rigid collars, compared with soft ones, contributed to significantly more immobilization in all directions including flexion/extension, lateral flexion to the right/lateral

Table 4. Comparison of cervical spine ROM wearing rigid collar and no collar using paired sample $t$-test

\begin{tabular}{lcccccc} 
Condition & Flexion & Extension & Rt. Lat. Flx & Lt. Lat. Flx & Rt. Lat. Rot & Lt. Lat. Rot \\
Rigid collar & $21 \pm 1.7$ & $33.7 \pm 2.8$ & $28.5 \pm 1.7$ & $28.6 \pm 2.0$ & $53.9 \pm 2.4$ & $53.5 \pm 2.0$ \\
No collar & $51.2 \pm 2.6$ & $62.9 \pm 3.2$ & $41.5 \pm 1.4$ & $41.3 \pm 1.3$ & $64.2 \pm 2.1$ & $64.2 \pm 1.6$ \\
$p$-value & $<0.01$ & $<0.01$ & $<0.01$ & $<0.01$ & $<0.01$ & $<0.01$ \\
\hline
\end{tabular}

Values are presented as mean \pm standard deviation.

ROM, range of motion; Rt. Lat. Flx, right lateral rotation; Lt. Lat. Flx, left lateral flexion; Rt. Rot, right lateral rotation; Lt. Lat. Rot., left lateral rotation.

Table 5. Comparison of cervical spine ROM wearing soft and no collar using paired sample $t$-test

\begin{tabular}{lcccccc} 
Condition & Flexion & Extension & Rt. Lat. Flx & Lt. Lat. Flx & Rt. Lat. Rot & Lt. Lat. Rot \\
Soft collar & $31.3 \pm 2.7$ & $44.5 \pm 2.7$ & $34.9 \pm 1.6$ & $35 \pm 1.6$ & $56.8 \pm 1.3$ & $56.6 \pm 1.0$ \\
No collar & $51.2 \pm 2.6$ & $62.9 \pm 3.2$ & $41.5 \pm 1.4$ & $41.3 \pm 1.3$ & $64.2 \pm 2.1$ & $64.2 \pm 1.6$ \\
$p$-value & $<0.01$ & $<0.01$ & $<0.01$ & $<0.01$ & $<0.01$ & $<0.01$ \\
\hline
\end{tabular}

Values are presented as mean \pm standard deviation.

ROM, range of motion; Rt. Lat. Flx, right lateral rotation; Lt. Lat. Flx, left lateral flexion; Rt. Rot, right lateral rotation; Lt. Lat. Rot., left lateral rotation. 
flexion to the left, and rotation to the right/ rotation to the left. These differences may be due to the different materials of soft and rigid collars. This result is consistent with those of other studies $[3,10]$.

This study indicated that different cervical collars have different effects on neck motion. The immobilization on flexion (soft 39\% and rigid 59\%) was greater than that on extension (soft $29 \%$ and rigid $46.4 \%$ ), which could be due to the fact that the extensors of the neck are more powerful compared with the flexors in people aged 18-25 years. Our results are similar to that of previous studies $[4,9,11]$.

The results of the present study showed that flexion and extension of the neck were more immobilized when compared with lateral flexion and rotation while subjects were using cervical collars. It can be due to the absence of a counter to limit lateral flexion and rotation. This result was similar to those of previous studies $[3,7,9,10]$.

For whiplash injury to be treated, cervical collars should be used for an extended period of time [12]. Thus, immobilization should create minimum discomfort to the patient in daily activities [13]. Daily tasks typically require $30 \%-50 \%$ of the neck ROM [14-16].

Considering that $30 \%-50 \%$ of the neck ROM is required to perform daily activities, soft collars did not sufficiently limit the neck ROM. Neck immobilization by rigid collars was sufficient only in the flexion/extension. This finding was similar to those of previous studies $[10,17]$.

Flexion and extension are the most repetitive motions in the neck which could be limited by rigid collars by $59 \%$ and $47 \%$, respectively [14]. However, soft collars limited flexion and extensions by $39 \%$ and $29 \%$, respectively.

One limitation of the present study was the lack of recruitment of subjects in different age groups. Few studies have been conducted on people in different age group, and most studies have evaluated subjects of age 20-30 years. Since elderly people generally require cervical collars after whiplash injury, a study on older subjects should be conducted. Furthermore, this study examined healthy subjects; future studies should be performed on injured subjects. As the neck motions were separately examined in the current study, investigating neck motion concurrent with daily activities while using cervical collars should be a focus of further research. A lack of long-term effects of cervical collars was another limitation of the present study.

\section{Conclusions}

The data in this study showed that both soft and rigid collars limited head and neck motions. However, subjects experienced more immobilization in flexion/extension than in lateral flexion and rotation. Finally, rigid collars resulted in more immobilization in all directions compared with soft collars.

\section{Conflict of Interest}

No potential conflict of interest relevant to this article was reported.

\section{ORCID}

Kourosh Barati: 0000-0002-3243-1281

Mokhtar Arazpour: 0000-0001-6855-063X

Roshanak Vameghi: 0000-0002-7456-8008.

Ali Abdoli: 0000-0003-3502-3985

Farzad Farmani: 0000-0003-0503-2370

\section{References}

1. Styrke J, Stalnacke BM, Bylund PO, Sojka P, Bjornstig U. A 10-year incidence of acute whiplash injuries after road traffic crashes in a defined population in northern Sweden. PM R 2012;4:739-47.

2. Hsu JD, Michael JW, Fisk JR. AAOS Atlas of orthoses and assistive devices. Philadelphia, PA: Mosby Elsevier; 2008.

3. Whitcroft KL, Massouh L, Amirfeyz R, Bannister GC. A comparison of neck movement in the soft cervical collar and rigid cervical brace in healthy subjects. J Manipulative Physiol Ther 2011;34:119-22.

4. Lunsford TR, Davidson M, Lunsford BR. The effectiveness of four contemporary cervical orthoses in restricting cervical motion. J Prosthet Orthot 1994;6: 93-9.

5. Duc C, Salvia P, Lubansu A, Feipel V, Aminian K. A wearable inertial system to assess the cervical spine mobility: comparison with an optoelectronic-based motion capture evaluation. Med Eng Phys 2014;36: 49-56.

6. Ferrario VF, Sforza C, Serrao G, Grassi G, Mossi E. Active range of motion of the head and cervical spine: a three-dimensional investigation in healthy 
young adults. J Orthop Res 2002;20:122-9.

7. Zhang S, Wortley M, Clowers K, Krusenklaus JH. Evaluation of efficacy and 3D kinematic characteristics of cervical orthoses. Clin Biomech (Bristol, Avon) 2005;20:264-9.

8. Sandler AJ, Dvorak J, Humke T, Grob D, Daniels W. The effectiveness of various cervical orthoses. An in vivo comparison of the mechanical stability provided by several widely used models. Spine (Phila Pa 1976) 1996;21:1624-9.

9. Mosenkis R. Comparison of three cervical collars in restricting cervical spine motion. Moorestown, NJ: Jerome Medical; 2001 Feb 28. CITECH test report: \#490-421.

10. Johnson RM, Hart DL, Simmons EF, Ramsby GR, Southwick WO. Cervical orthoses. A study comparing their effectiveness in restricting cervical motion in normal subjects. J Bone Joint Surg Am 1977;59: 332-9.

11. Dvorak J, Antinnes JA, Panjabi M, Loustalot D, Bonomo M. Age and gender related normal motion of the cervical spine. Spine (Phila Pa 1976) 1992;17: S393-8.

12. Schnabel M, Ferrari R, Vassiliou T, Kaluza G. Ran- domised, controlled outcome study of active mobilisation compared with collar therapy for whiplash injury. Emerg Med J 2004;21:306-10.

13. Verhagen AP, Peeters GG, de Bie RA, Oostendorp RA. Conservative treatment for whiplash. Cochrane Database Syst Rev 2001;(4):CD003338.

14. Bennett SE, Schenk RJ, Simmons ED. Active range of motion utilized in the cervical spine to perform daily functional tasks. J Spinal Disord Tech 2002;15:30711.

15. Janes JM, Hooshmand H. Severe extension-flexion injuries of the cervical spine. Mayo Clin Proc 1965; 40:353-69.

16. Miller CP, Bible JE, Jegede KA, Whang PG, Grauer JN. The effect of rigid cervical collar height on full, active, and functional range of motion during fifteen activities of daily living. Spine (Phila Pa 1976) 2010; 35:E1546-52.

17. Carter VM, Fasen JA, Roman JM Jr, Hayes KW, Petersen CM. The effect of a soft collar, used as normally recommended or reversed, on three planes of cervical range of motion. J Orthop Sports Phys Ther 1996;23:209-15. 